\title{
Ruolo del Papilloma virus umano nella patogenesi dell'infertilità maschile
}

\author{
Andrea Garolla ${ }^{1} \cdot$ Francesco Muscianisi $^{1}$
}

Accettato: 3 luglio 2020 / Pubblicato online: 8 giugno 2021

(c) The Author(s) 2021

\section{Aspetti generali del Papilloma virus umano}

I Papilloma virus umani (HPV) rappresentano un gruppo di virus con DNA e tropismo strettamente epiteliale verso cute e mucose, in particolare quelle delle regioni ano-genitale e oro-faringea. Si conoscono circa 200 genotipi di HPV che vengono classificati in alto, medio o basso rischio, sulla base della capacità di promuovere o meno la carcinogenesi dei distretti epiteliali infettati. Lo spettro patologico HPVcorrelato consta dunque di manifestazioni sia benigne, quali verruche e condilomi di vario tipo, sia maligne, quali il carcinoma della cervice, della regione testa-collo, dell' ano e del pene. La via di trasmissione sessuale rimane la più rilevante, rendendo quella da HPV una delle infezioni sessualmente trasmesse più diffuse, con circa 6,2 milioni di nuovi casi all'anno negli USA [1]. Tuttavia, oggi si riconosce anche una via di trasmissione manuale-genitale e orale-genitale, attraverso contatti sessuali non penetrativi.

\section{Presenza di HPV nel liquido seminale}

L'infezione da HPV nel maschio può localizzarsi anche a livello del tratto seminale e studi recenti hanno riscontrato la presenza dei virioni di HPV nel liquido seminale. Tecniche di immunofluorescenza e ibridazione fluorescente insitu hanno confermato la presenza dei virioni di HPV a questo livello. Nel liquido seminale il virus può essere presente sia nelle cellule di sfaldamento che legato alla testa degli

$\triangle$ A. Garolla

andrea.garolla@unipd.it

1 Unità di Andrologia e Medicina della Riproduzione, Dipartimento di Medicina, Università degli Studi di Padova, Padova, Italia spermatozoi (Fig. 1), tramite il legame del glicosamminoglicano Syndecan 1 presente sulla regione acrosomiale degli spermatozoi con la proteina L1 del capside virale [2].

\section{HPV parametri seminali}

Nei pazienti affetti da infertilità idiopatica, è stata riportata un'elevata prevalenza dell'infezione seminale (fino al 20\%) rispetto ai soggetti fertili e alla popolazione generale. In questi soggetti, numerosi autori hanno messo in luce il frequente un riscontro di astenozoospermia, ovvero di una ridotta motilità spermatica. Inoltre, nostri studi hanno evidenziato che la presenza di HPV nel liquido seminale rappresenta uno stimolo immunogenico per lo sviluppo di anticorpi antispermatozoo nel liquido seminale. Anticorpi delle classi IgA e IgG sono stati riscontrati sugli spermatozoi di soggetti infetti, adesi alla regione acrosomiale, in corrispondenza dei siti di legame del virus. Si ritiene che la presenza di tali anticorpi sia in grado di indurre un ulteriore peggioramento della motilità spermatica [3].

\section{Implicazione di HPV nella fertilità naturale e assistita}

L'infezione seminale da HPV gioca pertanto un ruolo rilevante nella riduzione della fertilità maschile, sia spontanea che assistita. Uno studio che confrontava 226 coppie infertili ha mostrato che le coppie infertili con infezione seminale da HPV presentavano una riduzione del tasso cumulativo di gravidanze naturali e assistite e un aumento del tasso di aborti quando confrontate con coppie non infette [4]. Anche per quanto riguarda la procreazione assistita con donazione 


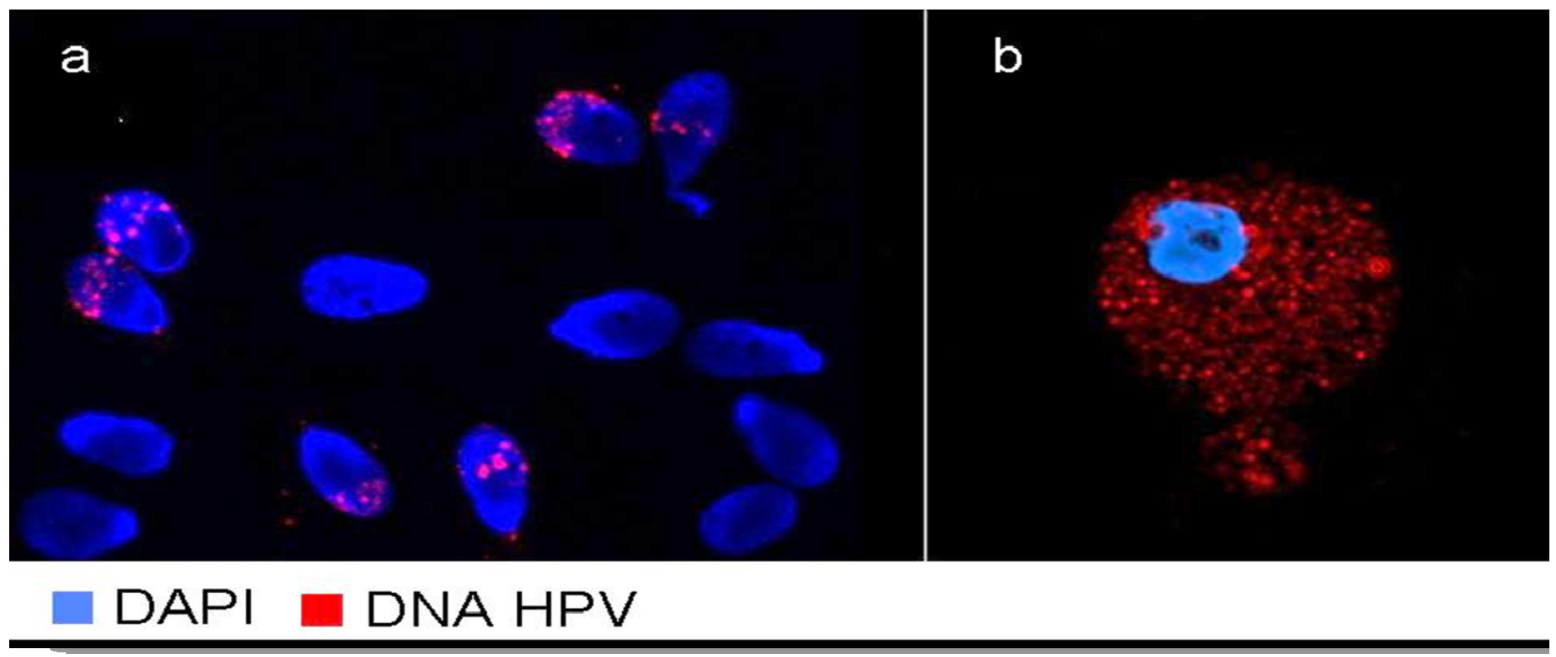

Fig. 1 Immunofluorescenza al microscopio confocale che mostra la presenza del DNA di HPV nella regione acrosomiale della testa degli spermatozoi (a) e all'interno del citoplasma di una cellula di sfaldamento uroteliale (b)

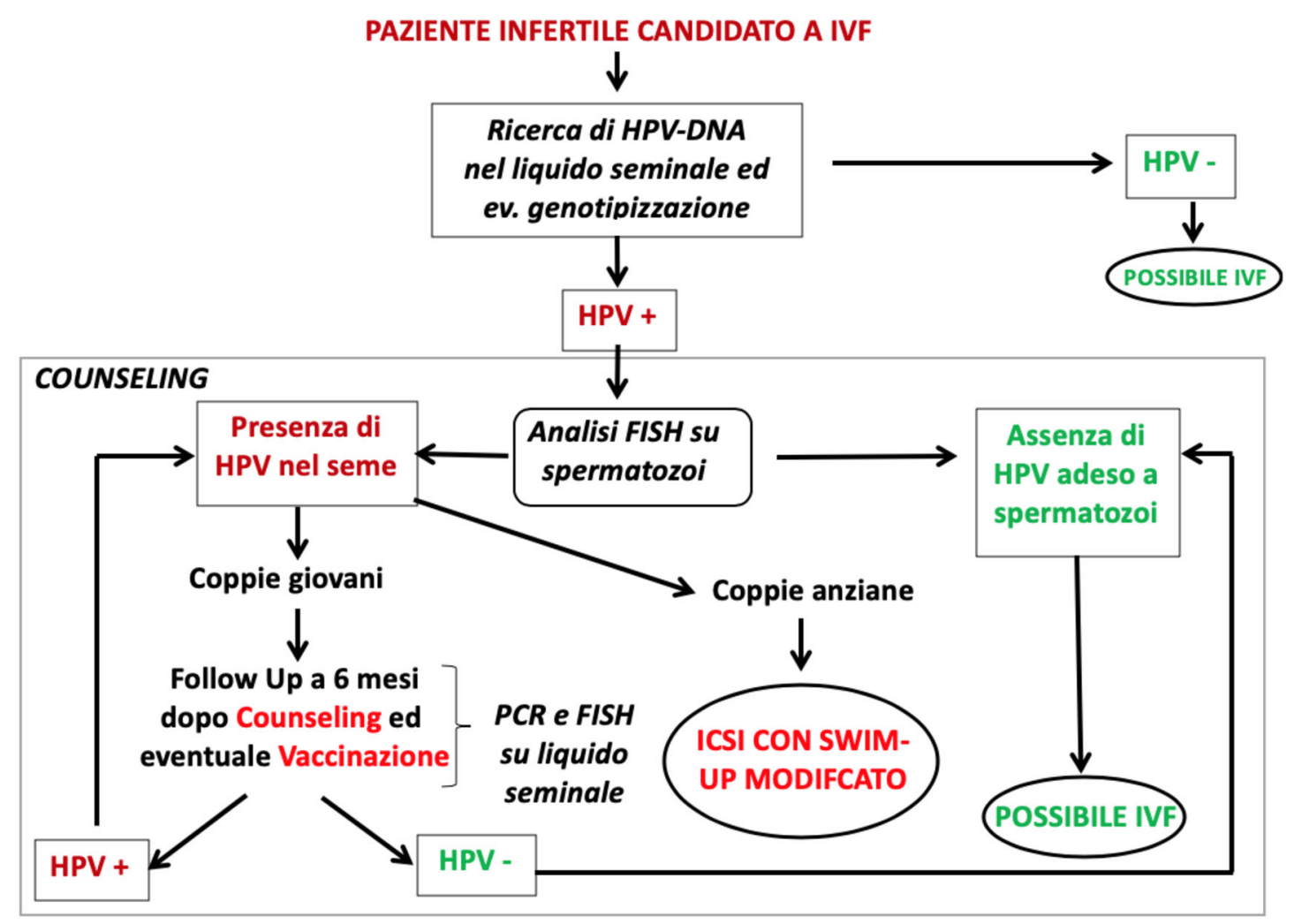

Fig. 2 Diagramma di flusso per la gestione dei pazienti infertili candidati a tecniche di procreazione assistita con infezione seminale da HPV. HPV, Human Papillomavirus; PCR, Polymerase chain reaction;

di spermatozoi, nel 2019 altri autori hanno riportato l'incapacità di ottenere gravidanze e anti nei casi di utilizzo di campioni seminali provenienti da donatori con seme infetto [5]. Non è ancora chiaro quali siano i meccanismi responsabili di questo fenomeno; tuttavia, alcuni studi in-vitro su
ICSI, Intra-cytoplasmic sperm injection; FISH, Fluorescent in-situ hybridization; $I V F$, In-Vitro Fertilization

cellule trofoblastiche transfettate con geni di HPV hanno dimostrato un aumentato tasso di apoptosi degli embrioni e una ridotta capacità di invasione della parete uterina. Questo processo potrebbe essere responsabile della degenerazione precoce del trofoblasto e, dunque, di aborto [2]. 


\section{Strategie per il miglioramento della fertilità nei pazienti infetti}

In assenza di una terapia efficace contro l'infezione da HPV, molteplici sono le strategie oggi utilizzate per contrastare l'infezione seminale nel maschio e migliorarne la fertilità.

\section{Counseling}

Uno studio del 2014 ha dimostrato, in coppie in cui almeno un membro era affetto da HPV, che il rispetto delle seguenti regole era in grado di ridurre la trasmissione e la persistenza del virus a livello seminale: 1) uso del preservativo; 2) attenta igiene del tratto riproduttivo e delle mani; 3 ) sospensione del fumo; 4) rimozione di eventuali lesioni condilomatose; e 5) uso di indumenti intimi e asciugamani strettamente personali e loro lavaggio a elevate temperature. In questo studio, le coppie che si attenevano strettamente al counseling avevano una significativa riduzione dell'infezione a 6 , 12,18 e 24 mesi di follow-up rispetto alle coppie infette di controllo [6].

\section{Vaccinazione adiuvante}

Secondo le ultime evidenze, l'utilizzo del vaccino con modalità adiuvante nei maschi con infezione seminale persistente da HPV sembra in grado di accelerare l'eliminazione del virus e produrre una stabile risposta anticorpale con una riduzione significativa del tempo medio di persistenza nel tratto seminale [7].

\section{Lavaggio del liquido seminale}

Il lavaggio del liquido seminale, attraverso uno swim-up modificato con l'utilizzo di enzimi proteolitici, si è rivelato efficace nella completa eliminazione del virus dalla superficie degli spermatozoi, senza alterarne significativamente le caratteristiche funzionali e l'integrità nucleare [2]. In Figura 2 è riportata una proposta per la gestione clinica dei pazienti infertili candidati a procreazione medicalmente assistita che presentano infezione seminale da HPV.

Funding Note Open access funding provided by Università degli Studi di Padova within the CRUI-CARE Agreement.
Conflitto di interesse Gli autori Andrea Garolla e Francesco Muscianisi dichiarano di non avere conflitti di interesse.

Consenso informato Lo studio presentato in questo articolo non ha richiesto sperimentazione umana.

Studi sugli animali Gli autori di questo articolo non hanno eseguito studi sugli animali.

Nota della casa editrice Springer Nature rimane neutrale in riguardo alle rivendicazioni giurisdizionali nelle mappe pubblicate e nelle affiliazioni istituzionali.

Open Access This article is licensed under a Creative Commons Attribution 4.0 International License, which permits use, sharing, adaptation, distribution and reproduction in any medium or format, as long as you give appropriate credit to the original author(s) and the source, provide a link to the Creative Commons licence, and indicate if changes were made. The images or other third party material in this article are included in the article's Creative Commons licence, unless indicated otherwise in a credit line to the material. If material is not included in the article's Creative Commons licence and your intended use is not permitted by statutory regulation or exceeds the permitted use, you will need to obtain permission directly from the copyright holder. To view a copy of this licence, visit http://creativecommons.org/licenses/by/4.0/.

\section{Bibliografia}

1. McQuillan G, Kruszon-Moran D, Markowitz LE (2017) Prevalence of HPV in adult aged 18-62: United States, 2011-2014. NCHS Data Brief 280:1-8

2. Garolla A, Lenzi A, Palù G et al (2012) Human papillomavirus sperm infection and assisted reproduction: a dangerous hazard with a possible safe solution. Hum Reprod 27(4):967-973

3. Garolla A, Pizzol D, Bertoldo A et al (2013) Association, prevalence, and clearance of human papillomavirus and antisperm antibodies in infected semen samples from infertile patients. Fertil Steril 99(1):125-131

4. Garolla A, Engl B, Pizzol D et al (2016) Spontaneous fertility and in vitro fertilization outcome: new evidence of human papillomavirus sperm infection. Fertil Steril 105(1):65-72

5. Depuydt CE, Donders GG, Verstraete L et al (2018) Time has come to include Human Papillomavirus (HPV) testing in sperm donor banks. Fact Views Vis Obgyn 10(4):201-205

6. Garolla A, Pizzol D, Vasoin F et al (2014) Counseling reduces HPV persistence in coinfected couples. J Sex Med 11(1):127-135

7. Foresta C, Garolla A, Parisi S et al (2015) HPV prophylactic vaccination in males improves the clearance of semen infection. EBioMedicine 2(10):1487-1493 